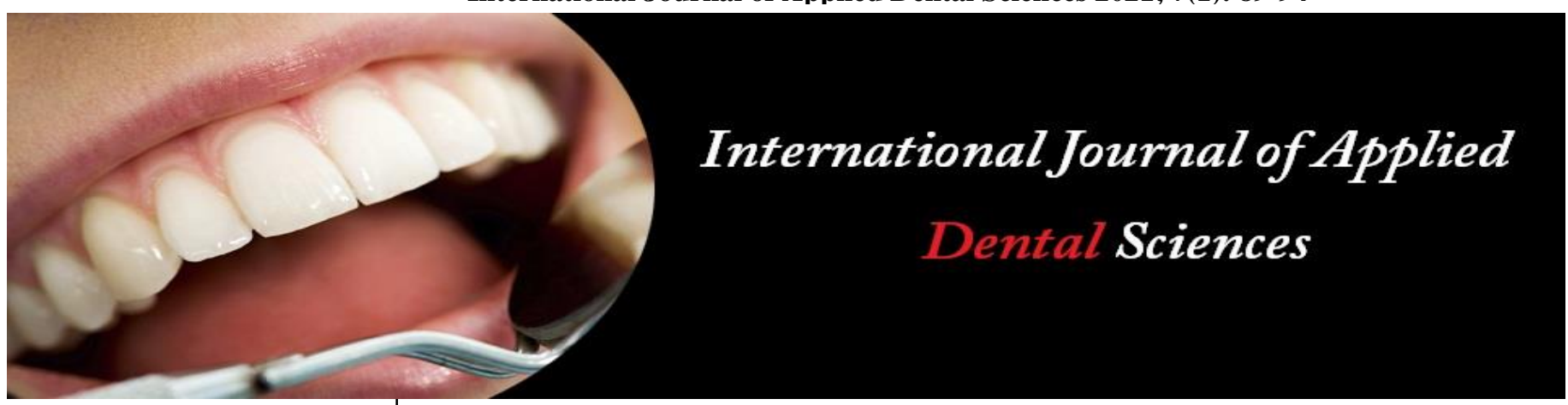

ISSN Print: 2394-7489

ISSN Online: 2394-7497

IJADS 2021; 7(1): 89-94

(C) 2021 IJADS

www.oraljournal.com

Received: 10-11-2020

Accepted: 12-12-2020

Snehanjan Sarangi

Clinical Tutor, Department of

Oral Pathology, Burdwan Dental

College and Hospital,

West Bengal, India
Corresponding Author: Snehanjan Sarangi

Clinical Tutor, Department of

Oral Pathology, Burdwan Dental

College and Hospital

West Bengal, India

\section{A brief insight on the etiopathogenesis of oral lichen planus}

\section{Snehanjan Sarangi}

DOI: $\underline{\text { https://doi.org/10.22271/oral.2021.v7.i1b.1121 }}$

Abstract

Oral Lichen Planus (OLP) is a relatively common, systemic, immune based disease. It usually, involves the oral mucosa, but other sites like the skin, genital mucosa, and scalp are also affected. In this autocytotoxic muco-cutaneous disease, the $\mathrm{T}$ lymphocytes trigger apoptosis of epithelial cells leading to chronic inflammation. OLP is mediated by both antigen specific and nonspecific pathways. Antigen presentation through basal keratinocytes and antigen-specific keratinocyte destruction by CD8+ cytotoxic T-cells are within the specific pathway spectrum. Whilst the nonspecific methods involve mast cell degeneration; leading to granular release and matrix metalloproteinase (MMP) activation. The process ultimately results in clustering of $\mathrm{T}$ cells in the upper layer of lamina propria, basal lamina disruption, $\mathrm{T}$ cell inflow within the epithelium, along with apoptotic death of keratinocytes. The normal oral mucosa may be an immune privileged site and breach of the immune barrier could result in OLP and possibly other autoimmune oral mucosal diseases owing to deficient antigen-specific TGF- $\beta 1$-mediated immunosuppression. However, many among these immuo pathologic mechanisms are still shroud in mystery.

Keywords: Oral lichen planus, pathogenesis, apoptosis, t lymphocytes

\section{Introduction}

OLP is a relatively common inflammatory disease, affecting between $0.5 \%$ and $2.2 \%$ of population ${ }^{[1]}$. Lesions can occur in oral, cutaneous and/or mucosal surfaces. The esophagus and conjunctiva are the less common sites of involvement. It mostly affects middle aged women ${ }^{[2,3]}$. This disease usually follows a course of chronic remission followed by a period of acute exacerbation.

\subsection{Variants of OLP and clinical repertoire in brief}

Generally, seven types of acknowledged oral manifestations of lichen planus include: (1) reticular, (2) papular, (3) plaque type (4) atrophic, (5) ulcerative (erosive) (6) vesiculo bullous form along with the desquamative gingivitis ${ }^{[4]}$. Skin lesions are purple, pruritic, polygonal lesions affecting the flexor surface of the extremities. Careful examination of surface of skin reveals lacy, fine white, radiating striae (Wickham stria).

\subsubsection{Reticular LP}

This is more common than the erosive form. There is characteristic patterns of interlaching white radiating lines; which may appear as papules. These lesions undergo periodic remission and exacerbation. Most commonly affected sites are buccal mucosa bilaterally, followed by lateral and dorsal tongue, gingival, palate.

\subsubsection{Erosive LP}

This form is not as common as reticular form. Clinically presence of atrophic, erythematous areas with central zones of ulceration of varying degrees can be seen. The peripheral zones of atrophic region are usually bordered by white, fine radiating stria. During examination, touching the area produces pain and bleeding. 


\subsubsection{Plaque LP}

It appears as an irregular, white, smooth plaque like area on the oral mucosa, predominantly involving the dorsum of tongue.

\subsubsection{Atrophic LP}

It has an erythematous background features intermediate between reticular and erosive LP.

\subsubsection{Bullous LP}

It consists of large bullae ranging in size from $4 \mathrm{~mm}$ to $2 \mathrm{~cm}$ which ruptures immediately.

Figure 1, 2 and 3 represent the clinical presentation of Reticular, atrophic and erosive OLP ${ }^{[5]}$.

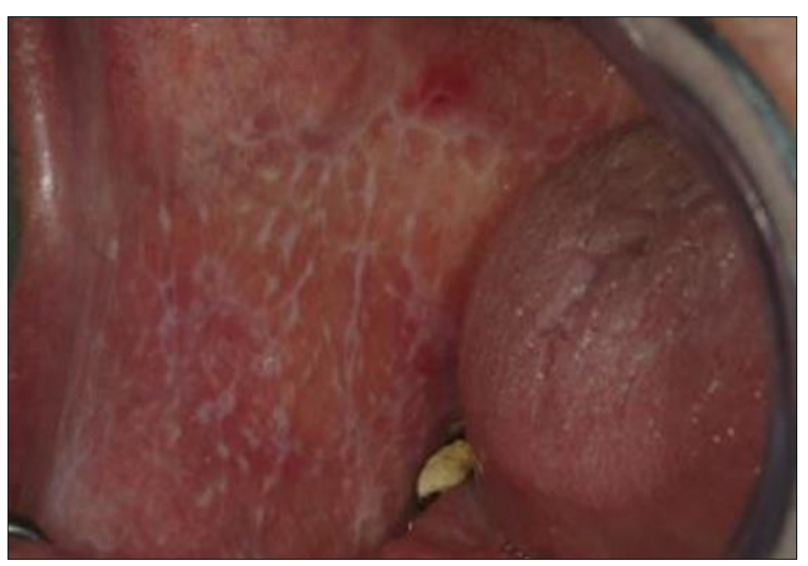

Fig 1: Reticular lichen planus involving buccal mucosa

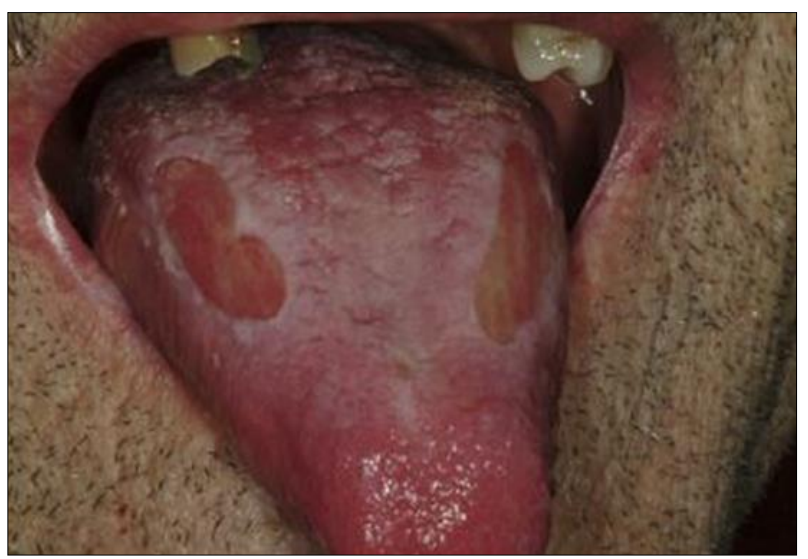

Fig 2: Atrophic LP on dorsum of tongue

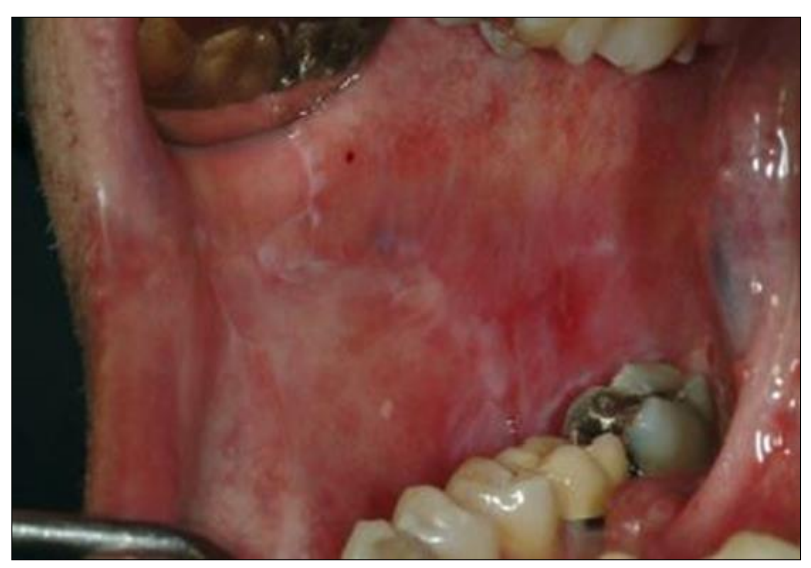

Fig 3: Erosive LP on buccal mucosa

\subsection{The etiologic factors}

Initiating LP are still not completely clarified. After much research in the immune pathologic mechanisms that underlie OLP, it is possible to place together part of a complex picture of the disease process. There appears to be a mechanism through which the regional cellular immune responses are activated and another for the $T$ lymphocyte response ${ }^{[6]}$ that culminates in the destruction of deep layered keratinocytes. The apoptotic death of basal level keratinocytes is augured by cytotoxic CD8 T cells ${ }^{[4]}$.

The amplified manufacturing of T helper (TH1) cytokines is a significant occurrence in LP, which is genetically dictated and controls the event of LP occurring in oral mucosa only; (interferon-gamma (IFN-Y)) dependant) or spanning the intraoral mucosae and dermal surfaces skin (tumor necrosis factor-alpha (TNF- $\alpha$ ) dependant) ${ }^{[7]}$.

The stimulated $\mathrm{T}$ cells are then tempted by intercellular adhesion molecules (ICAM-1 and VCAM), and they move towards the oral epithelium, further enhanced by Basement membrane proteins, such as collagen types IV and VII, ${ }^{[8]}$ laminins, integrins, and probably via CXCR3 and CCR5 mediated mechanisms ${ }^{[9]}$. Cytokines secreted by keratinocytes such as TNF- $\alpha$ and interleukins (IL)-1, IL-8, IL-10, and IL-12 are also chemotactic for lymphocytes. The $\mathrm{T}$ cells then bind to keratinocytes and IFN- $y$, and subsequent up regulation of $\mathrm{p} 53$, matrix-metalloproteinase 1 (MMP1) and MMP3 ${ }^{[10]}$ lead to programmed death of cells (apoptosis) ${ }^{[11]}$ of basal cells. The eukaryotic transcription factor nuclear factor kappa B (NF$\mathrm{k} \beta$ ), ${ }^{[12]}$ and the hindrance of the transforming growth factor mechanism (TGF-beta/smad) might clinically manifest as white lesions owing to increased multiplication of keratinocytes ${ }^{[13]}$. There have been sporadic reports of LP transforming to malignancy, especially the erosive and atrophic types ${ }^{[14]}$.

\subsection{Systemic disease involvement in LP}

Systemic diseases have been found to be in conjunction with LP; among them pathogenecity caused by hepatitis $\mathrm{C}$ virus (HCV) leads to extra hepatic signs -LP is amongst them.

$\mathrm{T}$ cells particularly, identified in $\mathrm{HCV}$, might play a pathognomonic role in formation of OLP ${ }^{[15]}$.

\subsection{Stress}

Increased severity of oral lichen planus has been associated with psychological stress and anxiety. Ivanovski et al. has opined that prolonged emotional stress can cause psycosomatization leading to a state of initialization and clinical appearance of OLP ${ }^{[16]}$.

\subsection{Tobacco chewing}

Daftary et al. have reported the presence of OLP like white, linear, wavy, and non-elevated streaks in a population of Kerala in an epidemiologic study of pre malignant and malignant diseases.

\subsection{Genetics}

It plays an important role in occurrence of OLP. Lowe et al. have first reported several cases of increased levels of HLAA3 in a group of British patients with cutaneous LP ${ }^{[17]}$.

\section{Immuno pathogenesis of OLP}

There are certain barriers in oral mucosal immune mechanisms, specifically pertaining to a lichenoid response. The phenomenon is illustrated by a delayed type hypersensitivity reaction, leading to apoptosis of the basal keratinocytes, orchestrated by CD8 T lymphocytes, which can be mediated a plethora of external stimuli, such as medicines, 
allergens, dental materials and stressful condition ${ }^{[18]}$. The exact causes, are not however clearly elucidated.

The mechanisms involved in the aetio-pathogenesis of OLP are multifactorial and likely to be synergistic:

1. Antigen-specific cell-mediated immune response

2. Loss of tolerance evidenced by the development of auto antibodies against self-antigens and the promotion of autoimmunity

3. Role of the humoral immune response

4. Non-specific immune mechanisms; and

5. Genetic factor.

\subsection{Antigen specific cell mediated immune response}

The initial triggering antigen causing the autoimmune response of OLP is undetermined. An endogenous peptide, bearing a protein sequence similar to the basal keratinocyte, might be responsible. Hence, OLP might be designated as an auto-immune condition. The probable outside mediators in OLP, like the dental materials, specific type of drugs, viruses and traumatic events exacerbate the self-antigens, or, might change the designated innate peptide sequences, resulting in their recognition by the immunecompetent cells as "non-self, that is "foreign". The resultant immune response develops in three stages: (1) T-cell migration within the epithelium, (2) Tcell activation, and (3) induction of basal keratinocyte apoptosis.

\subsubsection{T-cell migration into the epithelium}

Certain mechanisms have been postulated for interpreting the event. The "chance encounter" theory indicates that the resident, CD8+ cytotoxic T-cells invade the epithelium for regular immune vigilance but occasionally might face the presumed basal epithelial antigen. Also, the cytotoxic T-cells might be instructed by the keratinocytes, through the generation of cytokines, to percolate within the epithelium, resulting in lymphocytes to settle down involving antigencontaining basal keratinocyte, which is the "directed migration "postulate ${ }^{[19]}$.

\subsubsection{T-cell activation}

T-cells constitute the chief component of the lymphocytic conglomeration. The initiated cytotoxic CD8+ T cells comprise the predominant population among $\mathrm{T}$ cells in close approximation of deceased basal keratinocytes. Lymphocytes and other immune competent cells accumulate in an area of the maturing lichen planus augured by the discharge of cytokines. The helper $\mathrm{T}$ cells within lamina propria trigger the CD8+ $\mathrm{T}$ cells. Helper CD4+ $\mathrm{T}$ cells may be actuated by antigen derived from Class II MHC, being presented by the Langerhans cells, and through the keratinocytes ${ }^{[18]}$. Enhanced expression of Langerhans cells in lichen planus along with demonstration of up-regulated Class II MHC involvement is seen. Interleukin-12 (IL-12) produced by Class II MHC displaying Langerhans cells, and keratinocytes initiates production of interleukin-2 (IL-2) and interferon- $\gamma$ (IFN- $\gamma$ ) [19]. These cytokines (IL-12, IL-2 and IFN- $\gamma$ ) might be responsible for regulation of apoptotic death of keratinocytes. Within the OLP lesion a cascading occurrence of selfinducing and propagating T-lymphocyte activation is seen ${ }^{[20]}$. The entire gamut of these antigen specific cell mediated immune response orchestrations can be demonstrated by Figure $4^{[4]}$.

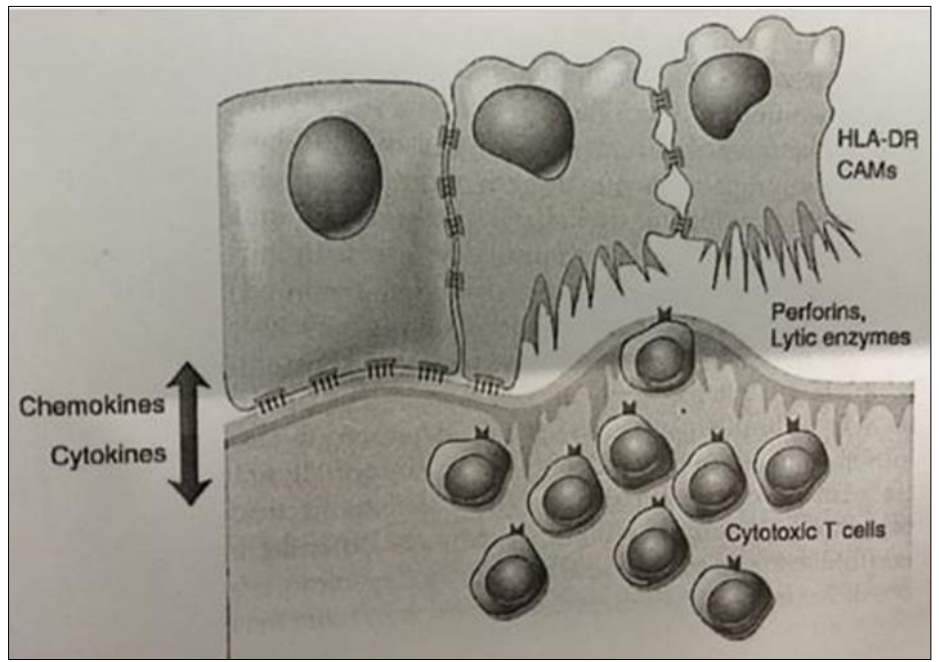

Fig 4: Antigen-specific mechanisms in OLP include antigen presentation by basal keratinocytes and antigen-specific keratinocyte killing by CD8+ cytotoxic T-cells. Non-specific mechanisms include mast cell degranulation and matrix metalloproteinase (MMP) activation in OLP lesions. These mechanisms may combine to cause T-cell accumulation in the superficial lamina propria, basement membrane disruption, intraepithelial T-cell migration, and keratinocyte apoptosis in OLP

\subsubsection{Heat-shock proteins}

In a study by Sugerman et al, an increased expression of Heat shock protein (HSP) within OLP basal/lesional keratinocytes was evident. Previous presence of an inflammatory process might precipitate HSP exhibition by keratinocytes. The pathophysiology of OLP might be dictated by an increased expression of HSP by LP lesional keratinocytes; this process is implemented by a myriad of extraneous agents (Medicines, trauma, infectious agents) ${ }^{[21]}$.

Auto antigenecity might be found within keratinocytic HSP in lichen planus lesions ${ }^{[21]}$ Impaired HSP gene expression by stressed oral keratinocytes might lead to increased propensity of OLP. This scenario is a plausible one, especially in the context of the lowered immune surveillance of OLP subjects. Thus, few instances of OLP lesions turning to malignancy, in reality might be an initial lichenoid response to previous neoplastic cells showing high HSP.

\subsubsection{Basal keratinocyte apoptosis}

Highly functional, CD8+ T-cells augur the programmed cell death of keratinocytes belonging to stratum basale in case of OLP lesions. In a dermatological study, T cells procured from OLP subjects were highly potent and had markedly high cytotoxic activity ${ }^{[22]}$. 
Cytotoxic CD8+ $\mathrm{T}$ cell mediated apoptosis of keratinocytes can take place via three well defined mechanisms: The first pathway, states that tumor necrosis factor- $\alpha$ (TNF- $\alpha)$, released by $\mathrm{T}$ cells, adheres to TNF- $\alpha$ receptor 1 upon the keratinocytes surface leading to their apoptosis ${ }^{[23]}$. The next mechanism deals, with the adherence of CD95 (Fas), upon the surface ligand of $\mathrm{T}$ cell, CD95L (Fas ligand), on the keratinocyte surface. The apoptosis of specified cells are mediated by Fas ligand (FasL), which is a variant of transmembrane protein. The third mechanism, opines that the keratinocytes are transfused with Granzyme B and Perforin (discharged from cytotoxic $\mathrm{T}$ cell and NK cell), leading to their apoptotic death.

\subsection{Perforin and granzyme induced apoptic damages}

Perforin, a protein, being composed of a polymeric complex (granzyme B, perforin, granulysin) is released by Cytotoxic T cells. They choose and gain entry to the susceptible keratinocytes via the mannose 6-phosphate receptor pathway. The Granzyme B, a serine protease, is then gradually discharged and initiates the apoptotic pathway leading to cell death, through a plethora of cascading events such as the breakdown of caspases (mainly caspase-3), which then promotes activation of caspase-activated DNAase, a DNA degradative enzyme ${ }^{[24]}$.

\subsection{Development of autoantibodies against self-antigens and the promotion of autoimmunity}

OLP, an immune based disease of complex etiopathogenesis, is characterized through the prolonged nature and chronicity of the process, frequency of occurring in middle age females, along with illustration of the T-cell auto reactivity as well as enhanced cytotoxicity directed towards basal keratinocytes, which are perceived to be foreign by immune surveillance and the successful implementation of immunosuppressive treatment modalitilies. Certain hypotheses are put forward to elucidate them like-(1) Paucity of Transforming Growth Factor- $\beta 1$ (TGF- $\beta 1$ ) leading to deregulated immune suppression; and (2) deprivation of "immune-privilege" in OLP.

\subsubsection{Paucity of transforming growth factor- $\beta 1$ (TGF- $\beta 1$ )}

TGF- $\beta$ plays a significant role in the immune surveillance through enhancing the differentiation of Forkhead box P3 (scurfin) and immune regulatory $\mathrm{T}$ cells (Tregs).

Scurfin acts as an orchestrator in the production along with functionality of $\mathrm{T}$ regulatory cells. TGF- $\beta$ might inhibit the stimulation of lymphocytes as well as the phagocytes. Reduced TGF- $\beta 1$ levels might be due to several factors: decreased amount of TGF- $\beta 1$ discharged by T regulatory cells (Th3); blockage of TGF- $\beta 1$ secretion; secretion of defective, non-functional TGF- $\beta 1$; faulty genetic expression of TGF- $\beta 1$ receptor ${ }^{[25]}$.

\subsubsection{Deprivation of "immune-privilege" in OLP}

The invading $\mathrm{T}$ lymphocytes might undergo programmed cell death in the epithelia of the oral mucosa, indicating that the oral epithelium enjoys an immune advantage alike the ophthalmic and placental regions. Here, the apoptosis pertaining to permeating inflammatory and immune regulatory cells that show Fas (CD95) is mediated by the stromal cells. $\mathrm{T}$ cell destruction can be augured by keratinocytes via the release of TNF $\alpha$ cytokines, through adherence to TNF receptor 1 on $\mathrm{T}$ cell surface.

Langerhans cells may also contribute to the loss of self- tolerance. Langerhans cells phagocytose the apoptotic bodies and debris of basal keratinocytes, but in doing so, may process and present to the CD4+ T helper cells a self-antigen derived from the remains of the basal keratinocyte. In turn, this may activate self-reactive CD4+ T cells that differentiate into Th1 or Th2 phenotypes and promote cell- or antibodymediated autoimmune reactions against basal keratinocytes, including the stimulation of the cytotoxic $\mathrm{T}$ cells against the basal keratinocytes ${ }^{[25,26]}$.

\subsection{Role of the humoral immune response}

The humoral immune response is thought to have some role in the pathogenesis of OLP, even though it is dominated by the T-cell-mediated immune response. Circulating auto antibodies to desmoglein 1 and 3 have been identified, but again the exact role of such auto antibodies remains uncertain.

The application of Rituximab, an anti CD 20 monoclonal antibody, leading to complete remission of a lichen planus case, owing to its action directed towards target B lymphocytes, asserts the significance of humoral immune responses in OLP ${ }^{[27]}$.

\subsection{Non-specific immune mechanisms}

Many T cells in lichen planus are nonspecific. Presence of a previous inflammatory process might trigger the migration of aforementioned $\mathrm{T}$ lymphocytes within epithelial layer, resulting in keratinocytic apoptosis. The processes involved are: (1) disintegration of basement membrane; (2) up regulated expression pertaining to matrix metalloproteinase (MMP); (3) Chemokine (C-C motif) ligand 5 (CCL5) (formerly called RANTES - Regulated on Activation, Normal $\mathrm{T}$ cell Expressed and Secreted) activity; and (4)Increased activity and degranulation of Mast cells.

\subsubsection{Basement membrane disruption}

The keratinocytes potentiate the stability of the epithelial part of the basement membrane, through releasing collagen IV and laminin $\mathrm{V}$ within basement membrane domain, although a reciprocal signal is also necessary to hinder the keratinocytic apoptotic degeneration. The cytotoxic CD8+ T-cells leads to impairment of immune surveillance mediated through keratinocytes, disintegrating basement membrane, and allowing the nonspecific $\mathrm{T}$ cells to mitigate within the epithelium. This in turn causes keratinocytic apoptosis, owing to deprivation of basement membrane mediated impulse. The process might interpret the chronic nature of lichen planus ${ }^{[27]}$.

\subsubsection{Matrix metalloproteinase (MMPs)}

MMP 9 content is usually up regulated in LP subjects as compared to the normal ones. Matrix metalloproteinases comprise a group of proteinases, possessing zinc as a component within them.

Collagen IV is broken down by MMP 9 and 2; connoted as gelatinases conjugatively. Tissue inhibitors of metalloproteinase (TIMPs), can hinder the matrix metalloproteinases by producing cross linked polymers accompanying MMPs or pro MMP's. MMP 9 stimulators are discharged by $\mathrm{T}$ cells, leading to basement membrane disintegration ${ }^{[28]}$.

\subsubsection{Mast cell}

Enhanced concentration of Mast cells in lichen planus, were found in a study performed by Sugerman et al. A spectrum of pro inflammatory initiators like TNF- $\alpha$, chymase, tryptase are found due to the discharge of Mast cell components [29]. 
Endothelial leukocyte adhesion molecule 1 (ELAM-1), intercellular adhesion molecules (ICAM) and leukocyte adhesion molecules are exhibited owing to the influx of cytokines by degranulating mast cells and macrophages ${ }^{[30]}$. MMPs are released as quiescent proenzymes and are rapidly destroyed after activation by activators like Chymase. Mast cell proteases along with MMP-9 released by $\mathrm{T}$ cells cause disintegration of basement membrane architecture ${ }^{[29]}$.

\subsubsection{Chemokine (C-C Motif) ligand 5 CCL5 (RANTES)}

Trigerred T-lymphocytes, oral keratinocytes and mast cells release CCL5, which plays a significant role in engaging of various immune and inflammatory cells, including lymphocytes, monocytes, eosinophils, basophils and mast cells. Mast cells, tempted by CCL5, degranulate releasing TNF- $\alpha$ and chymase, that cause increased production of CCL5, forming a cascading cycle, leading to chronic character of OLP ${ }^{[31]}$.

\subsubsection{Mast cell activation and degranulation}

Degeneration of the Mast cells leads to the release of a myriad of pre-inflammatory mediators, such as TNF- $\alpha$, chymase along with tryptase. CCL5 release through OLP T cells, might be mediated by Chymase and TNF $\alpha$, which might lead to further mast cell degranulation, causing the self-replicating cycle of apoptotic degeneration of cells ${ }^{[32]}$.

\subsection{Genetic factors}

The roles of genetic factors in OLP lesions play a pivotal role in the pathogenesis of the disease. Clinical presentation of OLP lesions might be attributed to the genetic variation of cytokines. Genetic polymorphisms of the first intron of the promoter gene of interferon- $\gamma$ and development of oral lesions of LP and an association between the -308A TNF- $\alpha$ allele and the development of cutaneous lesions of LP. The occurrence of OLP has also been linked to MHC class II allele DR6 in those patients who also have HCV-To date no specific HLA antigen profile has been found associated with idiopathic OLP [33].

\section{Conclusion}

OLP is a complex immune mediated disease where the etiopathogenesis is still shroud in mystery. The pathophysiology of OLP involves complex pathways. Antigen-specific mechanisms in OLP involve antigen presentation through basal keratinocytes and antigen- specific basal keratinocyte apoptosis by cytotoxic CD8 cells. Nonspecific mechanisms include mast cell degranulation and matrix metalloproteinase activation in OLP lesions. The initial triggering event in lichen planus lesions are yet to be clearly elucidated. Clearly further future research including detailed molecular, histopathological and immunological studies will help to shed more light on this complex topic.

\section{References}

1. Murti PR, Daftary DK, Bhonsle RB et al. Malignant potential of oral lichen planus: observation in 722 patients from India. J Oral Pathol 1986;15:71-77.

2. Carrozzo M, Gandolfo S. The management of oral lichen planus. Oral Dis 1999;5:196-205.

3. Eisen D. The clinical features, malignant potential and systemic associations of oral lichen planus: a study of 723 patients. J Am Acad Dermatol 2002;46:207-14.

4. Eversole LR, Sapp JP, Wysocki GP. Immune Mediated Disorders; Contemporary Oral \& Maxillofacial
Pathology. 2nd Edition, Mosby P257-258.

5. Scully C, Carozzo M. Oral mucosal disease: Lichen planus. British Journal of Oral and Maxillofacial Surgery 2008;46:15-21.

6. Scully C, Eisen D, Carrozzo M. Management of oral lichen planus. Am J Clin Dermatol 2000;1:287-306.

7. Carrozzo M, Uboldi de Capei, Dametto E et al. Tumor necrosis factor-alpha and interferon-gamma polymorphisms contribute to susceptibility to oral lichen planus. J Invest Dermatol 2004;122:87-94.

8. Eversole LR. Immunopathogenesis of oral lichen planus and recurrent aphthous stomatitis. Semin Cutan Med Surg 1997;16:284-94.

9. Ichimura M, Hiratsuka K, Ogura $\mathrm{N}$ et al. Expression profile of chemokines and chemokine receptors in epithelial cell layers of oral lichen planus. J Oral Pathol Med 2006;35:167-74.

10. Kim SG, Chae $\mathrm{CH}$, Cho BO et al. Apoptosis of oral epithelial cells in oral lichen planus caused by upregulation of BMP-4. J Oral Pathol Med 2006;35:3745.

11. Tanda N, Mori S, Saito K, Ikawa K et al. Expression of apoptotic signaling proteins in leukoplakia and oral lichen planus: quantitative and topographical studies. J Oral Pathol Med 2000;29:385-93.

12. Santoro A, Majorana A, Bardellini E et al. NF-kappa $\beta$ expression in oral and cutaneous lichen planus. J Pathol 2003;201:466-72.

13. Karatsaidis A, Schreurs O, Axell T et al. Inhibition of the transforming growth factor-beta/Smad signaling pathway in the epithelium of oral lichen. J Invest Dermatol 2003; 121:1283-90.

14. Bernard NA, Scully C, Eveson SW et al. Oral cancer development in patients with oral lichen planus. J Oral Pathol Med 1993;42:421-424.

15. Carrozzo M, Francia Di Celle, Gandolfo S. Increased frequency of HLA-DR6 allele in Italian patients with hepatitis $\mathrm{C}$ virus-associated oral lichen planus. $\mathrm{Br} \mathrm{J}$ Dermatol 2001;144:803-8.

16. Ivanovski $\mathrm{K}$, Nakova $\mathrm{M}$, Warburton $\mathrm{G}$ et al. Psychological profile in oral lichen planus. J Clin Periodontol 2005;32:1034-1040.

17. Lowe NJ, Cudworth AG, Woodrow JC. HL-A antigens in lichen planus. Br J Dermatol 1976;95:169-171.

18. Sugerman PB, Savage NW, Walsh LJ et al. Oral lichen planus: causes, diagnosis and management. Aust Dent J 2002;47:290-297.

19. Silverman S. Oral lichen planus: a potentially premalignant lesion. J Oral Maxillofac Surg 2000;58:1286-1288.

20. Farthing PM, Matear P, Cruchley AT. The activation of Langerhans cells in oral lichen planus. J Oral Pathol Med 1990;19:81-85.

21. Sugerman PB, Savage NW, Xu LJ et al. Heat shock protein expression in oral lichen planus. J Oral Pathol Med 1995;24:1-8.

22. Lodi G, Scully C, Carrozzo M et al. Current controversies in oral lichen planus: report of an international consensus meeting. Part 1. Viral infections and etiopathogenesis. Oral Surg Oral Med Oral Pathol Oral Radiol Endod 2005;100:40-51.

23. Dekker NP, Lozada Nur F, Lagenaur LA et al. Apoptosis associated markers in oral lichen planus. J Oral Pathol Med 1997;26:170-175.

24. Fricain JC, Sibaud V, Campana S et al. Mucosal 
pigmentation after treatment of oral lichen planus with topical tacrolimus. Dermatology 2005;210:229-232.

25. Zhang L. The regulation of Foxp3 expression in regulatory CD4 (+) CD25 (+) T cells: multiple pathways on the road. J Cell. Physiol 2007;211:590-597.

26. Yang LL, Liu XQ, Liu W et al. Comparative analysis of whole saliva proteomes for the screening of biomarkers for oral lichen planus. Inflamm Res 2006;55:405-407.

27. Lukac J, Brojovic S, Vucicevic-Boras V et al. Serum autoantibodies to desmoglein 1 and 3 in patients with oral lichen planus. Croat Med J 2006;47:53-58.

28. Zhou XJ, Sugerman PB, Walsh LJ et al. Matrix mettaloproteinases and their inhibitors in oral lichen planus. J Cutan Patho 2001;28:72-82.

29. Brew K. Tissue inhibitors of metalloproteinase: evolution, structure and function. Biochim Biophys Acta 2000;1477:267-8.

30. Regezi JA, Dekker NP, MacPhail LA et al. Vascular adhesion molecules in oral lichen planus. J Oral Surg Oral Med Oral Pathol Oral Radiol Endod 1996;81:682690.

31. Zhao ZZ, Sugerman PB, Walsh LJ et al. Expression of RANTES and CCR1 in oral lichen planus in association with oral lichen planus. J Oral Pathol Med 2002;31:158162.

32. Pigatto PD, Guzzi G. Oral lichenoid lesions: more than mercury. Oral Surg Oral Med Oral Pathol Oral Radiol Endod 2005;100:398-400.

33. Farhi D, Dupin N. Pathophysiology, etiologic factors, and clinical management of oral lichen planus, part I: facts and controversies. Clin Dermatol 2010;28:100-108. 